\title{
Les transformations et leurs dominantes : entre dynamique et structure de la processualité
}

\begin{abstract}
Abstrait
Ce texte traite méta-pédagogiquement les questions suivantes: (1) le dialogue, la rationalité des actions, la compréhension des traditions, de la liberté, du changement, des idéaux; (2) la transversalité, la dualité structurelle et les oscillations du développement; (3) les pièges, les contraintes et les lacunes des visions traditionnelles des relations entre les facteurs dans la transformation identitaire de l'homme; (4) une percée antipositiviste dans l'explication compréhensive des processus et un triple « effet explosif» dans les transformations de la représentation émotionnelle et cognitive des sujets agissants. Il aborde ces questions en référence à la psychanalyse, la réflexion critique et l'herméneutique.
\end{abstract}

Mots-clés : dominantes, dualité, dynamique, opérateur versus, structures, visions traditionnelles des processus, transformations.

\section{Transformations and Their Dominants: between the Dynamic and Structure of Processuality}

\begin{abstract}
The text discusses meta-pedagogically: (1) dialogue, rationality of actions, understanding of tradition, freedom, changes, ideals; (2) transversal, structural duality and developmental oscillations; (3) traps, limitations and gaps in traditional approaches to the transformation of human identity; (4) the anti-positivist breakthrough and the threefold "explosive effect" of the emotional-cognitive representation of actors. Psychoanalysis, critical thinking and hermeneutics are employed.
\end{abstract}

Keywords: dominants, duality, dynamique, operator versus, structures, traditional visions of processes, transformations.

\footnotetext{
* Académie de Poméranie à Słupsk, Pologne.

Article soumis le 2 février 2021 ; accepté pour publication le 11 mai 2021.
} 


\section{Introduction}

Depuis longtemps, nous essayons de «penser les transformations » de différentes manières, à l'image de ses variantes que nous pouvons observer dans la nature, l'environnement culturel, l'histoire, la vie sociale ou bien le développement individuel de l'être humain. Nous pouvons même observer la domination de nouvelles manifestations de ces processus de changement, tels que la globalisation ou les phénomènes probablement les plus dramatiques concernant l'époque anthropocène menaçant de destruction la civilisation et même la planète entière. Beaucoup d'entre eux ont une influence majeure sur les pratiques sociales, y compris les modèles de gestion des processus à l'échelle macro, les institutions et les procédures présentes dans les interventions professionnelles dans de nombreux domaines. Le mythe de l'époque de Lumières sur la linéarité du progrès n'existe plus, il y a bien longtemps on a formulé la règle de " croissance zéro » (growth zero) ou de "moratoire nucléaire», ou de durabilité et l'équilibre (sustainability) des processus. Tout cela devient indispensable du point de vue des opportunités de survie face aux multiples menaces provenant de l'ampleur de l'autodestruction et de l'autodégradation dans le monde.

Tout en sachant pouvoir transformer le monde en ruine civilisationnelle ou bien poubelle planétaire avec l'excès dans certains domaines et carences dans d'autres, on ne se soucie pas de développement durable (sustainable development). De plus, un grand nombre d'actions s'avèrent contreproductives. En effet, elles favorisent parfois des changements radicaux inverses aux escomptés, ou seulement déclarés, mais ayant leur propre vie sous forme des "programmes cachés » et nuisibles à différents niveaux ou échelles de résultats. De plus, la valorisation réflexive des processus est parfois décalée, dépourvue de l'aperçu de la « logique » réelle des changements qui s'opèrent. Il arrive souvent que l'on sous-estime leur importance ou les signes d'un attachement excessif à la thèse sur l'automatisme d'autorégulation et la capacité d'auto-correction. Parfois la transformation est empêtrée dans une dynamique de «boule de neige » en dehors de tout contrôle, il est impossible de l'arrêter sans changement radical de l'imagination, des habitudes, des procédures.

De façon générale, dans les interventions sociales, on ignore souvent les crises imminentes, les catastrophes générées, et mêmes les déficits les plus douloureux ou bien les erreurs graves. Les impacts n'y sont pas analysés à temps, ni soumis à la réflexion, ni corrigés. Les complexités non reconnues peuvent se venger, surprendre par leurs résultats contraires à la rationalité des actions. De plus en plus souvent, dans le rituel quotidien de l'apparence sociale, d'urgence et d'instrumentalisation, commence à croître, en tant que cause et résultat, le processus d'atrophie spirituelle, accélérant «l'atrophie de l'espace pour la réflexion » (Baudrillard 1998 ; Sloterdijk 2014). 
Cela se produit avec la participation des institutions qui devraient servir à la réflexivité, comme les écoles, les églises, ou les institutions de vie démocratique, y compris des parlements qui souvent ne font que participer au spectacle électoral d'arithmétique ad hoc. La pandémie covid-19 n'est qu'un maillon d'une longue chaîne des processus de dégradation, d'auto-dégradation, de frustration, de peurs, de phobies d'un côté, et d'aveuglement, de fanatismes, de violences et de narcissisme de l'autre. Les processus de changements et leurs pathologies non seulement fuient tout contrôle, mais ne sont pas non plus l'objet de réflexion autocritique ni de correction de la part de ceux qui les intensifient inconsciemment et de façon égoïste; comme c'est le cas par exemple dans : les populismes, l'aggravation de contradictions et d'inégalités, ou bien le discours de haine.

Nous ne sommes pas suffisamment sensibles à la complexité des processus, leur déroulement paradoxal et leurs résultats qui exigent un changement de perception, celle-ci toujours trop superficielle, provisoire et locale. De plus, nous apprenons, lentement et dans les douleurs d'accouchement, à donner de nouvelles significations aux différents termes et à leur application dans la pratique, et aussi à les lier à la nouvelle normativité postulée. Cette normativité demande un grand travail de correction lors des pratiques d'interventions professionnelles. Les transformations peuvent requérir de la résistance, elles peuvent se confronter elles-mêmes avec la résistance, et même directement le blocage de l'inclination de changement ou bien le refus de coopération. Parfois, elles se manifestent par l'initialisation des pressions, qui changent les cours des choses avec leur propre orientation, presque leur inertie, auxquelles on essaie de faire face afin de favoriser un autre processus qui n'a pas la force de voir le jour. Voici des contextes dramatiques que l'on ne doit pas perdre de vue quand on " pense transformations » en théorie des pratiques sociales.

\section{Champ de professionnalité meta-pédagogique}

De toute la mosaïque de ce drame, il convient de prendre en considération surtout un champ doté d'applications justifiées larges. Le but de cet article est de montrer la nécessité d'intégrer des catégories constituant une base pour analyser des processus de transformations dans les contextes de pratiques professionnelles de caractère meta-pédagogique.

Dans ce champ, l'activité se réalise toujours directement envers (versus) l'autre en définissant cette relation non seulement dans les catégories d'objet (agir "sur»), mais aussi dans les catégories de sujet (agir "pour»), et bien sûr interactives (agir «avec»), qui construisent des situations ou des états de processus dans lesquels la relation envers qui/quoi on agit est primordiale. Parfois la condition d'une transformation réussie est de conduire ses acteurs non seulement à l'accepter mais aussi à l'appuyer et à concourir activement à son 
déroulement. Les pressions institutionnelles ou seulement un «apport de conscience » ne suffisent pas en face de la nécessité de s'élever à un nouveau niveau de réflexion et de responsabilité dans l'action. Et de même, il serait probablement indispensable de mobiliser les acteurs d'une transformation en cours à la soutenir avec persévérance et de façon autonome, en tant que sujets, dans leur attitude pour le futur. Il s'agit-là d'une nouvelle identité étant «un style de synthèse d'expérience » (Erikson, voir Witkowski 2015) où se croisent différents aspects et parties prenantes dans l'existence professionnelle du sujet en tant qu'objet « hybride» socio-culturel.

Les conditions indispensables pour y parvenir sont :

(a) la perception et la valorisation de sa propre condition du sujet,

(b) la construction de la conception de soi et de ses réserves symboliques,

(c) l'élargissement des compétences opérationnelles ainsi que du potentiel de ses moyens techniques et de communication,

(d) la prise de décision stratégique sur son sort en tant que trajectoire de l'existence et $\mathrm{du}$ fonctionnement dans le monde dans lequel on est dynamiquement impliqué (Witkowski 2010).

Tout cela reste en relation avec le souci pour le champ d'émancipation de soi-même dans la réalité de son implication, celle-ci peut prendre la forme de soumission/ subordination dans la structure des relations sociales (p. ex. l'enseignant, disciplinant les apprenants et ayant pour mission leur développement d'autonomie, est impliqué dans la structure hiérarchique, et subit des pressions qui de façon institutionnelle le privent de liberté d'activité professionnelle).

De fait, l'auteur s'intéresse tout particulièrement à l'horizon psychosocial de la transformation en relation à la pratique persuasive culturelle. Il faut donc se référer aux mécanismes d'influence et de résistance rencontrés, où la dynamique est dotée d'un enchâssement interne, des tensions structurelles, et évidemment des contreréactions néfastes à ces derniers, dans la gestion des processus. Des ensembles de catégories forment des «écrans de perception» (Edward Balcerzan) dans la visualisation conceptuelle ayant un impact sur la modélisation des invariants de l'expérience. Ceux-ci constituant aussi bien temporairement un processus donné que des sujets eux-mêmes à une certaine étape de création de leur identité ou de construction de la relation de coopération et d'évolution du potentiel de transformation. Les invariants dans l'activité sont actuellement et structurellement enracinés dans une pratique donnée jusqu'à ce qu'il n'y ait pas de rupture, de destruction interne ou bien de crise si importante qu'elle ne puisse être gérée par des moyens disponibles, ou réglée grâce à une rationalité dominante précédemment.

L'hypothèse majeure de nos réflexions est la conviction que les systèmes binaires de pôles opposés qui apparaissent alors ne peuvent pas être considérés comme indépendantes l'une de l'autre. Ceux-ci, entremêlés dans un processus de changement, sont dotés d'une structure couplant leur relation dans des tresses ou 
des nœuds, dans des relations de dynamique variable, se présentant sous forme d'oscillations bipolaires ou de fluctuations. Dans cette dynamique de liens, nous retrouvons la dialectique de durabilité (sustainability) dans le cadre des transformations en cours en tant que condition de contrôle et d'orientation du processus. C'est une condition permettant d'éviter une catastrophe fonctionnelle du système, un chaos, ou bien sa désintégration en un mécanisme unipolaire, éliminant du système le pôle opposé, paradoxalement indispensable pour soutenir la complexité de l'action ou bien l'intégrité du système.

Dans ce texte, nous sommes contraints de présenter succinctement l'approche mettant en valeur la complexité structurelle des processus souvent mal repérée au cours de l'intervention professionnelle. Nous montrons la dynamique de changement de la situation à travers la question des facteurs dominants, tels que besoins, intérêts, tâches, composantes et interrelations. L'auteur se réfère de façon ponctuelle aux tropes inscrits dans les courants et réflexions: herméneutique, critique, psychanalytique, anthropologique. Ces courants et réflexions, dans le projet de l'auteur, apparaissent sous le terme « humanités appliquées ». L'adoption de cette approche pour toute activité pédagogique requiert un souci majeur pour la virtuosité dans l'action, la passion dans l'engagement et un enracinement profond dans des espaces symboliques étant une sorte de «sol culturel » porteur de vie (Radlinska, Witkowski 2014). Sa transformation et l'inclusion dans le processus impliquant au moins une dyade de sujets peut influencer considérablement le déroulement des processus déclenchés.

La thèse de cet article est, entre autres, la suggestion que la transition dans la séquence de compréhension de l'ontologie des actions sociales, notamment: fonctions - structure - processus devrait être complétée par la transition épistémologique analogique dans la séquence : alternative - continuum (spectre) - dualité structurelle dans la dynamique de processus. Il est donc indispensable de prendre en considération «des liaisons doubles» en tant que relations et références complexes (Bachelard 1980) allant jusqu'à la menace de tomber dans les pièges de «double contrainte » avoisinant la schizophrénie (Bateson 1987 ; ang. double bind).

Ce processus de transition exige une profonde réflexion et une revalorisation des fonctions courantes de l'opérateur versus en tant que celui qui sensibilise à la compréhension de la complexité d'un système donné et qui est confronté à une paire de dimensions ou de facteurs. Ces facteurs pouvant être en conflit, en excès ou en déficit des équilibrages renouvelés constamment. Cela ne concerne que les systèmes d'équilibrage récurrents temporaires face à de nouveaux états de procès qui les influencent, y compris de nouveaux états des facteurs extérieurs. Cet opérateur est souvent mal employé dans la théorie et la pratique de l'action, y compris dans des écrits académiques, en tant qu'expression d'opposition, de négation ou d'alternative pour décider sans équivoque dans la situation de soi- 
disant nécessité d'élimination du second élément. Il semble important de prêter un regard sur les défis de problématisation réfléchie des transformations inscrites aussi bien dans le professionnalisme des métiers meta-pédagogiques (les persuasions culturelles) que dans la percée antipositiviste et de dualité, cette dernière analysée et décrite à part par l'auteur. Les thèses détaillées ont été développées dans les travaux publiés par l'auteur ces dernières années et sont cités en bibliographie. Dans ce texte, elles apparaissent en tant que feuille de route pour une problématique, ou bien le répertoire de ce que l'on peut passer sur l'écran des transformations et de ses enjeux qui devraient être traités plus sérieusement que ce que l'on a l'habitude de faire.

\section{Les boucles de rétroaction et les transpositions des dominantes - exemples}

De plus en plus souvent, on utilise les références théoriques des sciences humaines contemporaines (Erik H. Erikson, Hans-Georg Gadamer, Jürgen Habermas, Robert Merton) pour parler du phénomène de la translocation (translation, transposition) des facteurs dominants dans la structure de différents processus en tant que base de transformation dans leur déroulement. D'autres profils et structures de facteurs dominants peuvent s'y manifester également, c'est-à-dire ceux qui sont perçus en tant que les plus importants dans la trajectoire périodique des processus.

I. Le changement des relations entre les dimensions de ce qui est juste, différent, propre à soi, génère des différences dans les orientations du processus de dialogue, avec la transition possible de la domination du pouvoir de persuasion réduisant la différence, passant par la compréhension de la voix d'autrui, respectant la différence et finissant par la réflexion grâce à une rencontre avec une vision sortant du cadre des moyens précédents de se comprendre soi-même. Dans ce caslà, il est très important pour la transformation de se révéler des "préjugés non conscientisés » au sens de Gadamer. Le "programme latent» de telles stratégies admet dans le premier cas d'indiquer qui est le pire et qui refuse l'entente. Dans le deuxième cas, l'éloge de la tolérance peut être réduite à une indifférence latente et le refus de prendre sérieusement en considération. Dans le troisième cas, l'affirmation de la valeur de rencontrer l'autre peut être dégradée en un intérêt d'utilité provisoire, immédiate. Toutefois, une ouverture sur les transformations de longue durée exige « une adaptation créative » (Obuchowski 1985) sous forme de travail en faveur d' " une tâche lointaine », sans garantie de réussite.

II. Il est également possible, en référence à la théorie de l'action de communication selon Habermas (1971) et aux 'intérêts cognitifs' qui en font partie, de montrer la différence entre différents types de rationalité que l'on relève dans l'activité professionnelle et le changement qui est généré par cette activité. Cela 
se produit en fonction de la relation entre : la technicité de l'activité en termes de bonne gouvernance, la qualité de la pratique de communication et de la construction de relations dans la coopération, et la capacité de libérer la situation des limites, barrières et obstacles pour passer à une alternative.

A ce moment-là, nous avons affaire :

(1) à la domination de l'instrumentalité dans l'action professionnelle qui introduit la relation de pouvoir et de subordination,

(2) au consensus orienté vers les liens et la communauté d'accord, ou bien

(3) à une nouvelle qualité grâce à «l'effet de décentration»: inclusion d'un nouveau point de vue dans l'horizon de compréhension du processus (Witkowski 2009a).

La problématique de «rationalité du processus de formation » reçoit ici de nouveaux stimuli (Milerski, Karwowski 2016).

III. Il en est de même pour l'établissement de la dominante dans la structure de la stratégie des attitudes orientées vers la construction sociale du sujet, par exemple se rapportant à la compréhension de la tradition, à la conception d'une vision de la liberté, du type de changement désiré ou bien la définition de l'idéal du lieu pour une telle formation. Cela génère d'autres types de transformations : conservatrice, libérale, socialement radicale ou bien dialogique, fondée sur la décentration, où les mêmes dimensions acquièrent une nouvelle signification grâce au changement de son emplacement dans la relation entre eux. Par exemple, le modèle de l'homme " émancipé » sera une fois celui qui agit comme un apôtre de la vérité en vigueur, celle-ci exigeant une transmission et une exégèse ; une autre fois, ce sera un artiste se donnant le droit à la transgression dans des interprétations originales. Séparément, la formation doit servir à un engagement révolutionnaire et directement au changement de la structure du milieu social pour favoriser l'émancipation des autres. Enfin, il s'agit d'avoir accès aux réservoirs de textes du domaine de la culture en tant que patrimoine symbolique grâce auquel le lecteur reçoit des stimuli éducatifs pour son développement, pouvant le transposer au-delà d'un champ localement disponible, dans le mode de "désocialisation », trans-

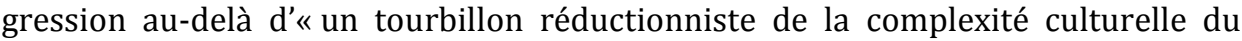
monde » de son propre lieu d'enracinement (Milan Kundera, Martin Heidegger; voir Witkowski 2007b).

IV. Le modèle paradigmatique de référence de la complexité qui se manifeste ici dans la structure des rôles sociaux (p. ex. de médecin, politicien, chercheur, éducateur de tous types) est le jeu de rôle dans le mode respectant d'ambivalence structurelle (ang. sociological ambivalence) entre les pôles de proximité et de distance (detached concern), entre donner l'orientation et suivre sous pression, ou bien entre le criticisme envers un repère extérieur et le contrôle autocritique de son activité (Merton, voir Witkowski 2007a). 


\section{Transversalité, oscillation, dualités}

Afin de penser à la qualité des processus d'intervention professionnelle, il est fondamental de souligner l'importance de l'approche transversale montrant les dualités structurelles dans des boucles de rétroactions. Nous avons ici des délibérations postulées également pour les champs d'activité dans les professions pédagogiques en pédagogie sociale (Marynowicz-Hetka 2019, 2020). Le fait de construire transversalement une gestion rationnelle face à la complexité des défis exige d'employer alternativement techniques et stratégies différentes tout en prenant en considération l'oscillation, dans le cadre des « esthétiques des situations éducatives » (Witkowski 2007a), entre :

- la relation du pouvoir : être «le premier » celui qui détient le pouvoir ;

- le déploiement de la force de sa personnalité séduisante ou charismatique : être «le deuxième », celui qui est significatif ;

- d'appel médiatique à la perspective de l'autrui en adoptant le rôle d'interprète : être « le troisième », catalyseur de la rencontre ;

- en fin de compte de création d'une situation d'acquisition de compétences pour coopérer sans intervenir soi-même: être «le quatrième» un animateur en retrait.

En effet, pour mener une action professionnelle et compétente, il ne s'agit pas d'opérer un choix entre les esthétiques données, modèles et formes d'agir et d'interagir, mais de savoir les utiliser par alternance et avec un déplacement de la dominante de style d'agir en fonction de la situation.

Ceci est possible dans l'approche intégrée de l'esthétique d'oscillations, pour laquelle chaque esthétique est dotée d'un double tranchant, étant source d'avantages et d'inconvénients, avec risque d'erreurs éthiques et stratégiques, donnant et reprenant, comme il en est avec toutes structures symboliques qui organisent notre activité (Anthony Giddens).

En arrière-plan, il s'agit également d'utiliser le modèle du cercle herméneutique comme indispensable pour décrire la situation de compréhension des complexités des relations bipolaires entre la partie et l'ensemble, entre le fait de dynamiser le processus et de maintenir la capacité d'équilibrer son déroulement, où la pratique de la réflexion et de correction de la situation de l'action est indispensable dans le mode de «retour incessant» pour réagir aux nouveaux contextes et conditions.

Il paraît que déjà la prise en considération de ces doubles systèmes, dans le processus de déclenchement de l'intervention professionnelle, permet de comprendre ses complexités dans chaque intervention sociale ainsi que l'évolution des sujets qui y sont impliqués. 


\section{Les doubles dominantes dans le processus de développement psychosocial}

Le fondement de l'approche adoptée par l'auteur est la lecture de la logique du processus de développement inscrite dans le modèle de cycle de vie d'Erikson qui nous permet, malgré sa vision réductionniste présentée dans de nombreux textes, de voir une structure complexe, double, des phases du processus de développement psychosocial avec des déplacements des dominantes évolutives (Witkowski 2015, 2020).

Elles marquent une tension majeure entre le pôle dynamisant le processus et le pôle stabilisant une étape du processus - cela demande bien sûr de les voir en oscillations conjointes et en coopération, et orientées différemment. Il est donc indispensable de produire des liens d'interdépendance contre les tentatives de fonder, dans une manière trop confortable, une univocité illusoire et réductionniste, unilatérale, une déconnection et une alternative. En fonction de la structure des liens entre les facteurs psychosociaux et le degré de leur intensité : méfiance, honte, culpabilité, dispersion/dissipation, retrait, stagnation, désespoir, nous voyons se créer une signification et valence, différentes, dans le processus de développement ne permettant pas de dynamiser le processus de développement ni d'intervenir pour favoriser ce développement.

$\mathrm{Ni}$ la négativité ni la positivité unilatérales ne permettent de dynamiser le processus de développement ni non plus d'intervention favorisant ce développement. L'orientation sur l'attitude dépourvue de coordination avec son contraire fonctionnel dans chaque cas de la phase, confiance, autonomie d'action, initiative, adéquation, identité, soins, création ou intégrité, génère l'abondance de certains facteurs ou le déficit d'autres. Le manque de connexions entre eux perturbe les processus d'équilibration des facteurs en tension et leurs déplacements conditionnant le développement.

En tant que modèle paradigmatique, pour les situations dont on parle ici, on pourrait évoquer la qualité de naviguer un yacht à voile de mer. La tension qui se produit entre la voile, exposition aux soufflements du vent étant en même temps une opportunité pour la navigation, et la quille, ou bien la qualité des stabilisateurs ad hoc de l'équipage, constitue un défi dans le processus de navigation en mer. Cette tension indique des changements fonctionnels indispensables, oscillations, fluctuations, alternances, pour coupler un ensemble complexe compris entre la dynamisation d'un mouvement et le soin pour sa stabilité dans un effort d'équilibration des avantages et des inconvénients, un risque et un éventuel succès (Witkowski 2020). 


\section{Les pièges des approches trop traditionnelles des relations entre les facteurs de processus}

Les approches traditionnelles dans la sphère de conceptualisation des processus de transformation et d'intervention professionnelle sont trop centrées sur les catégories des croisées de chemins, carrefours, alternatives, des pôles opposés des choix disjoints. Il est intéressant de découvrir les faiblesses de leur horizons et de construire des compléments afin de surpasser le cadre rétrécissant l'accès à la complexité. Cela est possible grâce à un lexique plus avancé, proposant une vision plus mûre des contextes de l'anthropologie, de l'ontologie ou bien de l'épistémologie des transformations des pratiques professionnelles (Barbier 2016, 2017). En même temps, dans ces approches traditionnelles les descriptions sont dominées par des affirmations des dichotomies en tant que dualités séparant, bifurcations ou polarisations qui sont tout au plus complétées par un spectre des potentialités en dispersion, décrites sur un continuum des gradations ou de hiérarchisation de séquences.

Pourtant, tout cela ne suffit pas face à la nécessité de reconnaître une complexité réelle à travers l'importance de voir des boucles de rétroaction, des dilemmes structurels, des enchevêtrements, faisceaux ou bien d'autres types de liaisons impliquées dans des tensions, conflits ou contradictions, mais qui se complètent réciproquement, et ayant pour elles-mêmes une fonction d'équilibre indispensable.

La vision d'un choix illusoire dans un dualisme perd de vue l'approche compréhensive du dilemme structurel en tant que couplage exprimé par un opérateur versus. Il celui-ci fonctionne en sensibilisant vers ce qui est difficilement unifié mais indispensable comme un accouplement des éléments opposés mais intégrés dans un système complexe. Il fonctionne sans déclencher un fractionnement facile et trop commmode, un antagonisme ou bien une désintégration éliminant l'un des éléments. Dans la structure des processus cet opérateur versus ne génère pas d'alternative, de disjonction ni d'opposition extrême, mais une tension créant, ou même exigeant, des conditions de coopération dans le cadre d'une stratégie fonctionnelle, celle-ci ne peut être menée à bien que par une unité nouvelle d'un niveau supérieur de la complexité.

Cela nous permet d'éviter les extrêmes, l'excès d'abondance et de privation, la déconnection et l'incompréhension des liens réciproques. Parmi les dichotomies nuisibles qui affectent la qualité des interactions éducatives et provoquent des difficultés dans l'activation des processus des transformations personnelles et identitaires, nous voyons perdurer, à cause des appauvrissements psychologiques, l'opposition des processus intellectuels et des phénomènes inscrits dans le sphère de l'émotion, du vécu et des implications. Dans des relations professionnelles, où le mécanisme de l'action est défaillant, perdure la 'ritualisation de l'apparence' (Pierre 
Bourdieu), l'indifférence et la distanciation affective qui bloquent par l'indifférence les processus d'identification et d'acceptation de l'importance des pulsions obtenus pour soi-même vers le développement.

En même temps cette approche traditionnelle dominante n'est pas capable d'inclure, dans sa compréhension des processus de transformation, l'opportunité d'opérer un bond, une discontinuité, une rupture avec l'ordre antérieur pour donner la place à une qualité nouvelle. Sur le plan des catégories d'anthropologie de pratiques professionnelles (Barbier 2016, 2017) il faudrait inclure dans la compréhension de processus de transformations un minimum nécessaire de références aux phénomènes tels que : rites de passage ou bien seuils d'initiation.

Il s'agit-là des aspects structurels des processus favorisant un nouveau type d'expérience dépassant les invariants précédents de la pratique et allant au-devant des nouveaux invariants qui prennent en considération la nouvelle étape identitaire comprise en tant que "style de synthèse de l'expérience" (Erikson). En même temps, cela demande de comprendre des barrières, des obstacles, des difficultés et des empêchements inscrits dans des "mécanismes de défense » inconscients, de plus, l'importance du refus de la structure de compétence précédemment dominante et la conception de soi (des définitions identitaires). Donc il n'est pas uniquement question de reconstruire un processus. Bien au contraire, il s'agit-là d'un travail de «déconstruction » d'un processus, d'ouverture vers une nouvelle réserve de conceptualisation, et d'essai de réorganisation structurelle, dans la quête d'une dynamique et des qualités nouvelles de fonctionnement.

Dans les contextes éducatifs cela signifie permettre la «désocialisation » qui, grâce au fait d'avoir quitté 'la réduction locale en spirale de la complexité culturelle du monde' (Kundera), donne la possibilité de mobiliser «l'effet de décentration » du développement (Habermas) dans le sillage de Jean Piaget. De là une nouvelle transformation ouvrant un horizon plus large aux interprétations où la diversité des points de vues disponibles devient un potentiel d'innovation et source d'un nouveau répertoire de rôles sociaux ainsi que de créativité dans "le jeu de rôles" post-conventionnel (Habermas, après Lawrence Kohlberg) sans suivre uniquement les scénarios prévus pour un rôle.

Bien sûr, les activités post-conventionnelles et leur demande, ainsi que l'ampleur des «structures innovantes » qui permettent leur manifestation, peuvent de différentes manières faciliter ou empêcher les transformations de l'espace de la pratique et de l'acquisition des compétences professionnelles (Przyborowska 2003). Et plus particulièrement, dans les processus de transformation et de leur stimulation, ce sont des «compétences de médiation » qui acquièrent une grande importance dans les pratiques professionnelles, p. ex. dans l'espace du travail social (Grudziewska, Lewicka-Zelent 2015). 


\section{Défis de la percée antipositiviste dans l'explicitation compréhensive d'un processus}

Il est indispensable aussi d'élaborer une certaine disponibilité à participer aux processus pour lesquels il n'y a pas d'accès préalable aux connaissances, permettant tout le contrôle du processus qui demande une intervention, ni non plus d'accès à une procédure réussie, illusion/ espoir positiviste. Il se manifeste alors une nécessité d'agir qui permet une collecte réfléchie des connaissances partielles, situationnelles, pouvant générer localement des invariants du champ disponible d'expérience, tout en veillant à «corriger les prémisses d'action » (Barbara Skarga), c'est-à-dire à apprendre sur ses propres erreurs servant de réorganisation et de reconceptualisation fondamentale du point de départ, par nécessité d'adopter une perspective antipositiviste de compréhension de l'activité/ de la pratique professionnelle.

Le vecteur épistémologique de la conscience méthodologique positiviste : « savoir pour anticiper $\rightarrow$ anticiper pour agir » doit être remplacé par le vecteur paradoxal de vision antipositiviste des relations entre l'action et les savoirs : « agir pour savoir $\rightarrow$ savoir pour corriger les prémisses d'agir $»$.

Cela signifie que, si l'on arrive à utiliser les réflexions de Gaston Bachelard, que le «réalisme » cognitif normalement postulé lors de l'activité doit être lié à la conscience d'être emmêlé/ impliqué dans des attitudes parfois suffisantes et parfois même dangereuses provenant de différents niveaux de compréhension de la situation du sujet.

Ce sont des approches réalistes du type :

(1) naïf dépourvues de difficultés supposant une clarté diagnostique et facilité d'accès ;

(2) instrumental recherchant un appui pour paramétriser réduisant les qualités aux quantités ;

(3) de solidarité conceptuelle se consolidant dans un recueil du lexique avec différents degrés de perception de ses limites à la pleine satisfaction ;

(4) d'ouverture conceptuelle supposant une complexité même de ce que l'on considère comme élémentaire dépourvu des paradoxes internes ou de tensions ;

(5) réaliste de "dispersion » qui ose construire une nouvelle approche à partir des attitudes dépourvues de référence théorique, qui se prête à être couplée et cohérente, entrant dans le cadre d'une conceptualisation pouvant servir d'une nouvelle stratégie de construction.

Nous savons bien que pour la compréhension finale des processus d'action, de connaissance et de transformation, en leur sein et entre eux, il faut admettre avec toute la maturité antipositiviste que les théories produites dans ces situations-là peuvent être perçues en tant que « représentation conceptuelle hypothétique des invariants d'expérience» (Enriques 1906). Ces dernières peuvent être niées en raison des retournements possibles, ruptures, pressions et faiblesses adaptatives, 
ou du moins peuvent faire voir les limites de leur champ d'application, occasionnant des catastrophes (Thom 1980, 1991), ou bien une phase aiguë, conformément au sens médical du phénomène de crise où l'on s'attend à un changement brusque du déroulement du processus de la maladie. Cela contraint à se manifester ou à faire une révolution en exigeant une nouvelle interprétation au niveau des facteurs fondamentaux et leurs relations ainsi que les modes de compréhension et d'attribution de signification.

Du fait de l'introduction originale par Habermas dans la pensée épistémologique de la catégorie de « l'intérêt cognitif » qui, en fonction de la technicité d'agir, la qualité de la pratique de communication et du champ d'émancipation, désigne d'autres types de rationalité (comme instrumentalle, consensuelle et decentrative), il faudrait réfléchir sur l'utilité de développer la catégorie «l'intérêt de transformation ». C'est possible par rapport aux phases particulières d'un processus en cours de réalisation tout en soulignant la thèse qu'il est soumis aux relocalisations dans le cadre de la dominante par/de phase dont la structure doit être appréhendée en tant que double en chaque moment. Il semble que c'est de cette manière que l'on devrait interpréter "l'intérêt de développement » de l'homme dans le modèle du cycle de la vie proposé par Erikson, ce sujet a été soulevé par l'auteur dans d'autres analyses (Witkowski 2015).

\section{Préoccupation pour « l'effet explosif » dans la transformation}

Dansle cadre des processus engagés professionnellement dans l'espace des professions variés de persuasion il faudrait prêter une attention particulière à l'enjeu d'attirer la disponibilité (motivation, les efforts, les soins) des acteurs de l'interaction, y compris les destinataires de l'intervention. Il faut savoir les attirer $\mathrm{au}$ « souci de soi » et à utiliser « les techniques de soi » (Michel Foucault) ou bien les «anthropotechniques» (Sloterdijk 2014). Lors de ces interactions, il est important de mobiliser des mécanismes triples "vécu - éveil - transformation » (Hesse) déclinant une triple gamme de «l'effet explosif » : destruction de l'évidence, ouverture des yeux, auto-acquisition. Tout cela conditionne la qualité de la présence réelle dans le processus en tant que 'disponibilité pour s'impliquer dans la rencontre' (Jacques Maritain) où la rencontre signifie 'le vécu d'une communauté d'expérience' (Gadamer) dans un type convenu de «justesse de l'attitude normative », en tant que réclamation de validité (validity claim) selon Habermas (Witkowski 2009a). Comme nous le savons, cet accord est parfois impossible avec des définitions divergentes de la propre vie par les destinataires du processus d'interaction, notamment dans les catégories d' "être en chemin ». Zygmunt Bauman propose des types suivants : pèlerin, vagabond, touriste, flâneur ou joueur. Les attirer vers l'interaction impliquée en tant que condition d'un processus réussi devient difficile et même impossible en raison d'écarts. 
La condition d'amener le destinataire des interventions professionnelles à une expérience existentielle pertinente (Mircea Eliade) est souvent un essentiel «vécu d'initiation ». Donc, l'effet explosif est indispensable pour détruire l'étape antérieure en tant qu'expression de naïveté, de manque de disponibilité, d'imagination sur ce qui va se produire en surmontant le seuil suivant. Il est nécessaire de détruire les évidences superficielles, les persuasions profondes en tant qu'impliqués ; ou encore des défaites non conscientisés, ou l'attitude de ne pas faire face à la vie en général. C'est pourquoi il est important de créer des espaces pour «la pédagogie du vécu ». Dans le deuxième aspect de mobilisation du processus de formation en tant que transformation, il est indispensable d'ouvrir de façon explosive l'accès à une nouvelle perspective, un nouveau point de vue, donc l'ouverture à une expérience que le destinataire n'aurait pas trouvé lui-même sans l'impulsion de l'autre.

Cet effet explosif à part de détruire les invariantes précédentes ouvre aussi le chemin d'accès aux sphères inaccessibles de l'inconscience humaine, mais aussi aux champs de la culture symbolique en tant que « milieu invisible » (Helena Radlińska, voir Witkowski 2014). C'est donc l'explosion qui ouvre les yeux à un nouveau type de réflexion qui est rendue possible et inscrite dans le type de " pédagogie d'éveil ». Cet éveil devient alors un terme paradoxal désignant un niveau important de qualité du processus d'intervention souvent présent dans différents types de narrations. A présent, il doit être inclus dans l'anthropologie des pratiques professionnelles.

Et enfin, le maillon intégral du processus de formation, en tant que transformation, est la mobilisation du processus de «transformation intérieure » chez le destinataire. Il faut «donner à penser» pour que certains stimuli symboliques soient perçus et transformés pour devenir des éléments internes d'un nouveau champ et niveau d'identité. Ils peuvent fonctionner en projetant sur la condition existentielle, sur les conceptions de soi et sur la qualité des compétences à agir. C'est une composante de l'explosion de transformation qui dans le contexte de la crise identitaire mène au paradoxe d'une «nouvelle naissance » (Erikson).

En s'imprégnant du "discours d'autrui », en accumulant et transformant des "perles de penser » symboliques l'homme a un accès plus profond/approfondi à la compréhension et expression de soi-même (Mikhail Bachtin, voir Witkowski 2000).

\section{Sur l'importance de l'alliance interactive}

La qualité d'orientation univoque d'un processus, peut s'entremêler à un refus de coopération même au niveau de générer une identité négative (Erikson, voir Witkowski 2015). Celle-ci décleche le processus de s'identifier avec les contenus opposés aux persuadés, non malgré les efforts, mais à cause de leur incapacité 
d'établir «une alliance de développement» concernant le souci de soi. Comme nous le savons, « une alliance psychothérapeutique» (Witkowski 2020) est une condition intégrale pour tout effort d'aide thérapeutique, mais on ne prend pas en considération les soucis analogiques pour la condition de cet accord dans d'autres champs d'implication et de l'expérience professionnelle. Il est donc indispensable de proposer une nouvelle vision du professionnel en tant qu'impliqué dans l'espace cognitif, émotionnel et de compétence du destinataire des interventions, s'approchant vers l'interaction qui doit servir à ce dernier d'entreprendre le rôle subjectif personnellement motivé dans le souci de soi.

Cette vision a pour objectif de promouvoir le style de la synthèse de l'expérience professionnelle à partir de la position d'un «intellectuel transformatif» (Henry A. Giroux, voir Giroux, Witkowski 2010) qui comprend que sa tâche consiste à faire construire un potentiel d'autonomisation (empowerment) en tant que capacité à se revendiquer dans les interactions sociales et à « s'émanciper » des limites venant de l'inconscient du destinataire, y compris des liens au professionnel lui-même dans le mode d'être psychanalytiquement reconnu en tant que «transfert ». Les actions institutionnelles peuvent, d'un côté, démunir de subjectivité à travers « la privation du courage civil» pour être autonome et critique, et, de l'autre côté, endommager cette subjectivité à travers des mécanismes de séduction et d'attachement, par la fascination de la personne du professionnaliste et son pouvoir d'influence.

Dans les catégories d'autorité, aussi bien dans une relation éducative que dans le processus de transformation personnelle, cela signifie la nécessité de remplacer les mécanismes interactivement comfortables : autoritarisme d'assujettissement, autorité comme garantie d'infaillibilité ainsi qu'autorisation formelle de passer aux phases suivantes du processus par exemple par promotion à la classe supérieure de l'école. Il est donc nécessaire de conduire le destinataire à coopérer de sa position avec « l'autorité à ses pieds » qui désigne davantage le phénomène, déjà décrit par l'auteur, "d'auto-rite de passage», renouant avec l'inspiration anthropologique où l'effort de transformation demande de s'enraciner dans la culture en tant que "sol symbolique » pour arriver, en confrontation autonome avec le professionnel et ses inspirations, à l'accroissement du potentiel subjectif au sens de l'émergence d'une disponibilité (capacité, motivation, outils) à grandir dans l'activité personnelle pour relever les défis.

\section{Limites du caractère transformatif d'impact}

La dominante processuelle inscrite dans une phase de l'activité professionnelle ne peut pas être seulement la pratique de transmission unilatérale ou à sens unique des contenus sans se soucier de leur signification pour le récepteur dans interaction, car les contenus doivent influencer de façon importante la qualité 
d'identité du destinataire à des conditions acceptables pour lui, si on l'inclut au processus de transformation de soi-même. En effet, il ne s'agit pas d'entreprendre une action professionnelle qui sert à encourager la transgression orientée vers la réalisation de soi-même. Il se passe ainsi surtout si l'individu est en état de crise identitaire et son potentiel de se définir est dispersé ou bien a été nié après la chute des invariants antérieurs d'expérience sur le champ nouveau de l'effondrement existentiel et de manque de motivation à rester dans les cadres de l'organisation précédente de la vie. Les effets transformatifs d'activité dépendent très souvent du degré de la détermination dans l'auto-transcendence (Tillich) de la reconstitution de soi-même.

Il en est de même surtout quand on voit se manifester des difficultés avec l'énergie de l'esprit mobilisant à la transformation de l'individu à se former de nouveau, ou bien débloquer son développement et se tirer de l'effondrement (Orzelska 2014). L'intervention professionnelle peut ne pas avoir de chances par rapport à l'individu restant sans énergie vitale à entreprendre de nouveaux essais au moment où il manque d'imagination, d'espoir et de détermination indispensables pour les efforts qui mobilisent de nouveaux processus de développement. Il n'est pas suffisant non plus d'avoir la dominante d'implication de transformation dans la motivation d'un professionnel à l'attitude de solidarité avec les autres si l'individu ne se débrouille pas avec lui-même.

L'activité professionnelle orientée vers la mobilisation des processus de transformation personnelle demande un changement de la dominante motivant et organisant cette activité par l'ouverture de l'espace de diversité et de complexité pour lequel le destinataire de l'intervention doit être compris et pris en charge dans ses propres actions. Le professionnel ne peut rien montrer dans le mode de transformation, il peut seulement aider à voir en mettant à disposition une autre perspective de se voir par l'acteur du processus orienté vers lui-même. Ce processus ne se manifestera pas si le destinataire ne s'y retrouve pas personnellement en tant que sujet de l'interaction au sein d'une nouvelle communauté d'engagement et en rompant avec la détérioration antérieure de sa condition et les dommages dans la capacité d'être soi-même.

Le terme «communauté» invite également à prendre conscience de la différenciation de ses niveaux d'interaction : en commençant par une communauté d'intérêt où la relation d'interaction est instrumentale, en passant par la communauté des tâches qui de façon fonctionnelle est mêlée aux règles très strictes, imposées en tant que minimum absolu, et en finissant par la plus difficile, dont le maintien demande des efforts incessants de valorisation, la communauté en tant que tâche impliquant participation autonome dans une affirmation de valeurs approuvées et vécues. Il s'agit-là des valeurs et des principes auxquelles elle veut servir tout en exigeant une attitude subjective et le respect de la subjectivité de chacune des parties participantes. 


\section{Transformation en tant que transformation de soi}

Une réelle rencontre avec l'autre devient une chance pour soi et uniquement de cette façon peut être mise à disposition afin de ne pas être rejetée, même si elle est transformée en dehors de l'horizon subjectif d'intentions du professionnel. Le processus de transformation obtient une nouvelle qualité du moment où il est capable, ou admet dans son cadre un méta processus de transformation de soi, de se libérer du projet initial, des cadres interprétatifs et de l'ensemble des moyens utilisés.

Aussi bien Witold Gombrowicz que Karol Wojtyła et Roman Ingarden nous sensibilisent, chacun dans le cadre de son domaine d'intérêt, par ses œuvres, ses conceptions des actes versus la personne et en remplissant les lacunes indéfinies dans la littérature, que c'est l'œuvre qui crée ... son auteur, comme l'acte peut créer la personne, et le texte vit dans la réception si nous lui transmettons un sens vivant et vital pour nous. L'anthropologie de la pratique professionnelle doit prendre en considération de telles circonstances et les inclure dans les postulats concernant la qualité des processus de transformation qui constituent bien des pratiques s'engageant dans le sort de l'autrui à travers le fait de lui attribuer une nouvelle place dans la culture et dans la vie sociale. Bien sûr, cela exige un effort pour munir le professionnaliste des compétences de transformer en soi des acquis de l'humanisme dans son acception intégrale et holiste des sciences humaines, de façon à ce que le processus d'intervention ouvre les espaces symboliques, mis à la disposition des intéressés en tant que chances à saisir. En général, la disponibilité à interagir dans cette direction n'est pas un fait donné en soi, donc le professionnel (enseignant, thérapeute, manager, ...) doit veiller à la construire avec une préoccupation.

Ce n'est pas en vain que Antonin Wagner a forgé, dans le contexte des barrières dans le travail social, le modèle de homo disoeconomicus, pour désigner les attitudes des destinataires de l'intervention professionnelle qui n'ont pas la capacité à se comporter rationnellement. Ils ne sont pas non plus prêts à assumer les charges ou surcharges, ne savent pas agir dans des conditions dépourvues de garanties de certitude, et de plus, sont pris en otage par le passé, ou bien, n'ont pas la capacité de s'ouvrir à une perspective longue pour la réalisation des tâches lointaines, ou bien encore, par exemple dans les cas de dépendances, ne savent pas valoriser l'appui dans la communauté à laquelle ils ont accès, ou même peuvent détruire cet appui en le considérant comme sans importance. Dans cette situation, bon nombre d'hypothèses concernant l'interaction professionnelle doivent faire l'objet d'un examen approfondi, car le manque de disponibilité à interagir ne nous libère pas toujours éthiquement de l'effort de transformation d'une telle attitude pour que le lancement du processus même d'amélioration soit possible. 


\section{Conclusion}

$\mathrm{Vu}$ le contenu restreint de cet article et donc l'impossibilité de documenter et d'illustrer pleinement les thèses présentées, l'auteur invite à les approfondir de la façon plus développée dans les livres cités (Witkowski 2010, 2013, 2014, 2015, 2018). Il est intéressant de souligner que l'approche proposée ici est de plus en plus souvent présente et analysée, indépendamment des références citées ci-dessus, dans des travaux réalisés par les auteurs à partir de différentes perspectives et différentes disciplines, comme la sociologie ou la philosophie. Ce qui est important c'est la vision des aspects de transformation en termes de transactions sociales, de conceptualisation d'un double l'entre-deux (in-between) dans le contexte de fluidité sociale (Foucart 2016), et certaines versions d'herméneutique mettant en valeur entre autres d'après Paul Ricoeur un équilibre instable en tant que type d'un état qui requiert toujours une transformation (Wierciński 2003, 2019). De même, en sociologie de l'éducation et théorie du travail social en Pologne, les références structurelles et dynamiques en termes de nouveau lexique en tant que base théorique et cadre méthodologique, comme dilemmes, dualité, enchevêtrement, tensions, constituent un axe d'analyses et d'essais d'application (Frysztacki 2019). En sociologie, le cadre le plus large pour ce type d'approche est probablement proposé par la réflexion dans les catégories du processus civilisationnel (Elias 2011). Alors il est clair, que les considérations de cet article devraient être prolongées dans l'avenir.

\section{Bibliographie}

Bachelard G. (1940) La philosophie du non. Essai d'une philosophie du nouvel esprit scientifique, Paris, Presses Universitaires de France.

Bachelard G. (1980) Le matérialisme rationnel, Paris, Presses Universitaires de France.

Bachelard G. (2000) Filozofia, która mówi nie. Esej o filozofii nowego ducha w nauce, trad. Justyna Budzyk, Gdańsk, Słowo/obraz terytoria.

Barbier J.-M. (2016) Leksykon analizy aktywności. Konceptualizacje zwyczajowych pojęć, trad. Ewa Marynowicz-Hetka, Łódź, Wydawnictwo Uniwersytetu Łódzkiego.

Barbier J.-M. (2017) Vocabulaire d'analyse des activités. Penser les conceptualisations ordinaires - Essai, Paris, Presses Universitaires de France.

Bateson G. (1987) Steps to an Ecology of Mind. Collected Essays in Anthropology, Psychiatry, Evolution and Epistemology, Extended Edition, London, Northvale. 
Baudrillard J. (1998) Ameryka, trad. Renata Lis, Warszawa, Wydawnictwo Sic!

Elias N. (2011) O procesie cywilizacji. Analizy socjo- i psychogenetyczne, trad. K. Markiewicz, T. Zabłudowski, Warszawa, Wydawca W.A.B.

Enriques F. (1906) Problemi della scienza, Bologna, Zanichelli.

Foucart J. (2016) Fluidité sociale et conceptualisations de l'entre-deux. Systèmes semichaotiques, réseaux et transactions sociales. Essai, Aix-en-Provence, Ēditions Presé.

Frysztacki K. (2019) Wokół pracy socjalnej. Od koncepcji i teorii do kontekstów empiryczno-analitycznych, Kraków, Wydawnictwo Uniwersytetu Jagiellońskiego.

Giroux H. A., Witkowski L. (2010) Edukacja i sfera publiczna. Idee i doświadczenia pedagogiki radykalnej, préface Z. Kwieciński, postface T. Szkudlarek, annexe : Z. Melosik, B. Śliwerski, Kraków, Oficyna Wydawnicza „Impuls”.

Grudziewska E., Lewicka-Zelent A. (2015) Kompetencje mediacyjne w profesji pracownika socjalnego, Warszawa, Difin S.A.

Habermas J. (1971) Knowledge and Human Interests: A General Perspective, Boston, Beacon Press.

Marynowicz-Hetka E. (2019) Pedagogika społeczna. Pojmowanie aktywności w polu praktyki, Łódź, Wydawnictwo Uniwersytetu Łódzkiego.

Marynowicz-Hetka E. (2020) Social Pedagogy. Comprehending Activity in the Field of Practice, trad. M. Hinton, Beau Bassin, Labert Academic Publishing.

Milerski B., Karwowski M. (2016) Racjonalność procesu kształcenia. Teoria i badanie, volume 2, Kraków, Oficyna Wydawnicza „Impuls”.

Obuchowski K. (1985) Adaptacja twórcza, Warszawa, Książka i Wiedza.

Orzelska J. (2014) W stronę pedagogiki istotnej egzystencjalnie. Życie i jego trudności $z$ energiq duchowa jako wyzwanie pedagogiczne rezyduów tożsamości, Kraków, Oficyna Wydawnicza „Impuls”.

Przyborowska B. (2003) Struktury innowacyjne w edukacji. Teoria - praktyka - rozwój, Toruń, Wydawnictwo Uniwersytetu Mikołaja Kopernika.

Sloterdijk P. (2014) Musisz życie swe odmienić. O antropotechnice, trad. J. Janiszewski, préface A. Żychliński, Warszawa, Wydawnictwo Naukowe PWN.

Thom R. (1980) Paraboles et catastrophes. Entretiens sur les mathématiques, la science et la philosophie, réalisés par Giuglio Giorello et Simona Morini, Milano, Il Saggiatore. 
Thom R. (1991), Parabole i katastrofy. Rozmowy o matematyce, nauce i filozofii, avec Giuglio Giorello i Simoną Morini, trad. et préface R. Duda, Warszawa, Państwowy Instytut Wydawniczy.

Wierciński A. (ed.) (2003) Between Suspicion and Sympathy. Paul Ricoeur's Unstable Equilibrium, Toronto, The Hermeneutic Press.

Wierciński A. (2019) Existentia Hermeneutica. Understanding as the Mode of Being in the World, Zurich, Lit Verlag.

Witkowski L. (2007a) Edukacja wobec sporów o (po)nowoczesność, Warszawa, Wydawnictwo Instytutu Badań Edukacyjnych.

Witkowski L. (2007b) Między pedagogika, filozofiq i kulturq̨. Studia, eseje, szkice, Warszawa, Wydawnictwo Instytutu Badań Edukacyjnych.

Witkowski L. (2009a) Ku integralności edukacji i humanistyki II. Postulaty - postacie - pojęcia - próby. Odpowiedź na Księgę Jubileuszową, Toruń, Wydawnictwo Adam Marszałek.

Witkowski L. (2009b) Wyzwania autorytetu w praktyce społecznej i kulturze symbolicznej (przechadzki krytyczne w poszukiwaniu dyskursu dla teorii), Kraków, Oficyna Wydawnicza „Impuls”.

Witkowski L. (2010) Tożsamość i zmiana. Epistemologia i rozwojowe profile w edukacji, Wrocław, Wydawnictwo Naukowe Dolnośląskiej Szkoły Wyższej.

Witkowski L. (2013) Przełom dwoistości w pedagogice polskiej. Historia, teoria, krytyka, Kraków, Oficyna Wydawnicza "Impuls".

Witkowski L. (2014) Niewidzialne środowisko. Pedagogika kompletna Heleny Radlińskiej jako krytyczna ekologia idei, umysłu i wychowania. O miejscu pedagogiki w przełomie dwoistości w humanistyce, Kraków, Oficyna Wydawnicza „Impuls”.

Witkowski L. (2015) Versus. O dwoistości strukturalnej faz rozwoju w ekologii cyklu życia psychodynamicznego modelu Erika Homburgera Eriksona, Kraków, Oficyna Wydawnicza „Impuls”.

Witkowski L. (2018) Humanistyka stosowana. Wirtuozeria, pasje, inicjacje. Profesje społeczne versus ekologia kultury, Kraków, Oficyna Wydawnicza „Impuls”.

Witkowski L. (2020) Psychodynamiki i ich struktura. Studia z humanistyki stosowanej, Toruń, Wydawnictwo Adam Marszałek. 


\section{Transformations and their Dominants: between the Dynamic and Structure of Processality}

\section{Summary}

The text discusses the so-called meta-pedagogical transformational contexts in the spaces of human activities and fate, with the use of references to the concept of hermeneutics, critical reflection, psychoanalysis, anthropology and the original project defined as "applied humanities". The author highlights the synthetic image of the considerations emerging from his main books.

The problem structure of the article: (1) the field of meta-pedagogical professionalism; (2) feedback and displacement of the dominant among the factors in professional processes and practices - examples: dialogue, rationality of actions, understanding of tradition, freedom, change, the ideal of the space of action (transmission, transgression, transformation, translocation); (3) transversality, structural duality and oscillations in the space of action dilemmas and phase dominants in the processes of psychosocial development; (4) traps, limitations and gaps of traditional approaches to the relationship between the factors in the transformation of a person's identity, losing discontinuities and internal tensions, and structural reorganization; (5) the challenges of the anti-positivist breakthrough in the status of theory as a representation of experience invariants and in an understanding explanation of processes, and the threefold "explosive effect" in the transformation of the emotional-cognitive representation of actors, including the importance of their self-transformation and interactive alliance in action; (6) other approaches to the indicated conceptualization deepening the issues.

The key terms of the text are: process dominants, paradoxes and limitations of transformation, duality, dynamics, the versus operator, factor displacement as structure reorganization, theory seen as anti-positivist, traditional approaches to processes. The following authors are the main references in the background literature to the text: Barbier, Erikson, Gadamer, Giroux, Habermas, Marynowicz-Hetka, Merton, Radlińska, Ricoeur, Sloterdijk, Wierciński, Witkowski.

\section{Transformacje i ich dominanty: między dynamiką i strukturą procesualności}

\section{Streszczenie}

Tekst omawia tzw. meta-pedagogiczne konteksty transformacyjne w przestrzeniach działań i losów ludzkich, z wykorzystaniem odniesień do koncepcji hermeneutyki, refleksji krytycznej, psychoanalizy, antropologii i autorskiego projektu określonego mianem „humanistyki stosowanej”. Autor uwypukla syntetyczny obraz rozważań wyłaniający się z jego głównych książek.

Struktura problemowa artykułu: (1) pole profesjonalności meta-pedagogicznej; (2) sprzężenia zwrotne i przemieszczenia dominanty wśród czynników w procesach 
i praktykach zawodowych - przykłady: dialog, racjonalność działań, rozumienie tradycji, wolności, zmiany, ideału przestrzeni działania (transmisja, transgresja, transformacja, translokacja); (3) transwersalność, dwoistość strukturalna i oscylacje w przestrzeni dylematów działania i dominant fazowych w procesach rozwoju psycho-społecznego; (4) pułapki, ograniczenia i luki tradycyjnych ujęć relacji między czynnikami w transformacji tożsamościowej człowieka, gubiące nieciągłości i napięcia wewnętrzne oraz reorganizację strukturalną; (5) wyzwania przełomu antypozytywistycznego $\mathrm{w}$ statusie teorii jako reprezentacji inwariantów doświadczenia i w rozumiejącym wyjaśnianiu procesów oraz troisty „efekt wybuchowy” w transformacjach reprezentacji emocjonalno-poznawczej podmiotów działających, w tym znaczenie ich autotransformacji i przymierza interakcyjnego w działaniu; (6) inne ujęcia wskazanej konceptualizacji pogłębiające problematykę.

Kluczowe terminy tekstu to: dominanty procesów, paradoksy i ograniczenia transformacji, dwoistość, dynamika, operator versus, przemieszczenia czynników jako reorganizacje struktury, teoria widziana antypozytywistycznie, tradycyjne ujęcia procesów. Główne odniesienia do literatury w zapleczu tekstu stanowią następujący autorzy: Barbier, Erikson, Gadamer, Giroux, Habermas, Marynowicz-Hetka, Merton, Radlińska, Ricoeur, Sloterdijk, Wierciński, Witkowski.

\section{Pour citer cet article}

Witkowski L. (2021) Les transformations et leurs dominantes : entre dynamique et structure de la processualité, „Nauki o Wychowaniu. Studia Interdyscyplinarne” 2(13), 66-87, https://doi.org/10.18778/2450-4491.13.06 